\title{
Mosaicism for mitochondrial DNA polymorphic variants in placenta has implications for the feasibility of prenatal diagnosis in mtDNA diseases
}

\author{
David R Marchington ${ }^{1}$, Martin Scott-Brown ${ }^{1}$, David H Barlow ${ }^{1}$ and Joanna Poulton ${ }^{* 1}$ \\ ${ }^{1}$ Nuffield Department of Obstetrics and Gynaecology, John Radcliffe Hospital, Oxford OX3 9DU, UK
}

Women who have had a child with mitochondrial DNA (mtDNA) disease need to know the risk of recurrence, but this risk is difficult to estimate because mutant and wild-type (normal) mtDNA coexist in the same person (heteroplasmy). The possibility that a single sample may not reflect the whole organism both impedes prenatal diagnosis of most mtDNA diseases, and suggests radical alternative strategies such as nuclear transfer. We used naturally occurring mtDNA variants to investigate mtDNA segregation in placenta. Using large samples of control placenta, we demonstrated that the level of polymorphic heteroplasmic mtDNA variants is very similar in mother, cord blood and placenta. However, where placental samples were very small $(<10 \mathrm{mg})$ there was clear evidence of variation in the distribution of mtDNA polymorphic variants. We present the first evidence for variation in mutant load, that is, mosaicism for mtDNA polymorphic variants in placenta. This suggests that mtDNA mutants may segregate in placenta and that a single chorionic villous sample (CVS) may be unrepresentative of the whole placenta. Duplicates may be necessary where CVS are small. However, the close correlation of mutant load in maternal, fetal blood and placental mtDNA suggests that the average load in placenta does reflect the load of mutant mtDNA in the baby. Provided that segregation of neutral and pathogenic mtDNA mutants is similar in utero, our results are generally encouraging for developing prenatal diagnosis for mtDNA diseases. Identifying mtDNA segregation in human placenta suggests studies of relevance to placental evolution and to developmental biology.

European Journal of Human Genetics (2006) 14, 816-823. doi:10.1038/sj.ejhg.5201618; published online 3 May 2006

Keywords: prenatal diagnosis; mitochondrial DNA; mtDNA segregation; heteroplasmic mtDNA polymorphisms; placental patch

Introduction

Mitochondria generate energy for all cellular processes. Their own maternally inherited chromosome, the mitochondrial DNA (MtDNA), encodes subunits of the respiratory chain. mtDNA diseases may be caused by mutations in

*Correspondence: Professor J Poulton, Nuffield Department of Obstetrics and Gynaecology, John Radcliffe Hospital, Oxford OX3 9DU, UK.

Tel: + 441865 221067; Fax: + 441865 769141;

E-mail: joanna.poulton@obs-gyn.ox.ac.uk

Received 13 May 2005; revised 13 February 2006; accepted 16 February 2006; published online 3 May 2006 either mitochondrial or nuclear genes and hence give rise to maternal or autosomal patterns of inheritance. In normal individuals, every cell contains thousands of identical copies of mtDNA (homoplasmy).

Both mutant and normal mtDNA are found in individuals (heteroplasmy) with mtDNA disorders, and this is important because the degree of heteroplasmy is a determinant of disease severity. ${ }^{1}$ Furthermore, the load of mutant mtDNA may vary widely between different tissues from a single patient. ${ }^{2,3}$ Accumulation of mutant mtDNA in specific tissues appears to underlie some instances of disease progression. ${ }^{3,4}$ Although both chorionic villous 
sample (CVS) and prenatal counselling are routinely available for Mendelian disorders, ${ }^{5,6}$ heteroplasmy prevents their routine use in mtDNA diseases. ${ }^{7}$ In addition, there is now evidence that in one case a proportion of inherited mtDNA was of paternal origin. ${ }^{8}$

The risk of transmitting a mtDNA disorder is difficult to assess $^{7}$ because the offspring of a heteroplasmic woman may vary widely both in their phenotype and in their mutant load. ${ }^{9,10}$ This is because of the so-called bottleneck in transmission of mtDNA. We and others have shown that a single woman may transmit markedly different levels of variant mtDNA to her oocytes, ${ }^{11,12}$ both for pathogenic and polymorphic mtDNA mutants. These and other data ${ }^{13}$ suggest that there may be a much smaller number of founder mtDNAs for each child than the $100000 \mathrm{mtDNAs}$ found in each normal oocyte. In other words, there appears to be a mitochondrial bottleneck. Logically, prenatal diagnosis of mtDNA disorders should be central to management, in practice it is fraught with uncertainty because of the bottleneck. ${ }^{14}$ Moreover, if there is regional variation in mutant load, ${ }^{2,3}$ a single blastomere or CVS may be unrepresentative of the load in the major part of the embryo. Further, if the correlation of mutant load with disease severity is poor, even a representative sample is of limited value. Moreover, changes in the distribution of mutant after the biopsy may invalidate the information. ${ }^{2}$ These difficulties appear to be so extreme that clinicians have suggested radical alternative management, for instance nuclear transfer techniques in an attempt to alter the mtDNA load of an embryo. ${ }^{15}$

The logistics of studying mtDNA segregation in a large number of placentae from families with mtDNA disease would be formidable. We have previously used naturally occurring polymorphic variants to study the bottleneck in human oocytes, ${ }^{11}$ and our inferences regarding both its timing and magnitude are consistent with data on pathogenic variants. ${ }^{12,16}$ In order to assess whether regional variation in the level of heteroplasmic mtDNA is likely to be a major feature of mtDNA transmission, we therefore sought naturally occurring heteroplasmy at polymorphic sites within mtDNA. Heteroplasmic polymorphisms are now held to be more common than previously supposed. ${ }^{17}$ Heteroplasmy frequently occurs at specific mutation hotspots such as nt16224, 16311 and 16093. ${ }^{18}$

In order to assess the feasibility of routine CVS in mtDNA disease, we compared the levels of heteroplasmic polymorphic variants between maternal blood or buccal DNA and placental levels and between placenta and cord blood. To determine whether a single sample is likely to be representative, we studied multiple samples from two placentae (of 7 and 40 weeks gestation) where we had identified two or more variants. We investigated the distribution of both heteroplasmic point mutations, and also length variation in a homopolymeric $\mathrm{C}$ tract centred around the thymidine at $\mathrm{nt} 310,{ }^{11}$ from multiple different samples of the same placenta.

\section{Materials and methods}

All of the control placentas and parental blood or buccal smear samples were obtained from normal controls with the approval of the Oxfordshire Research Ethics Committee from 18 mother, father, placenta trios from term pregnancies with informed consent. Cord blood was also available in 13 of these. Similarly, samples of chorionic villus were available from 16 therapeutic terminations of pregnancy at 7-12 weeks. Endometrium and chorionic villus were dissected apart in all cases. We studied multiple samples of one of the 16 therapeutic terminations and one term placenta. In these cases, samples of all types of material available were taken including cord, membranes and different depths and regions of the chorionic plate. Parental samples were sequenced to identify differences in hypervariable region one (from 16050 to $16350 \mathrm{bp}$ ) in the 18 trios. For convenience, we have named the site changes A-F (see Table 1). Because parental samples other than endometrium were not available from the 16 therapeutic terminations, we investigated only length variation around 310 bp (length variants named short, medium and long, corresponding to the modal length and one base pair shorter or longer, Table 1). T-PCR for heteroplasmic length variation $^{11}$ and solid-phase mini-sequencing for common heteroplasmic polymorphisms were carried out as described previously. ${ }^{19}$ Both methods quantitate the proportion of heteroplasmic variants in a specified region of around $1-3 \mathrm{bp}$. The detection primer for nt16069 (site D) was 5'ACCACCCAAGTATTGACT3', for nt16093 (site C) was 5'TCAACAACCGCTATGTAT3', for nt16224 (site A) was 5'AAGTACAGCAATCAACCC $3^{\prime}$, for nt16189 (site E) was 5'ATCCACATCAAAACCCCC3', for nt16256 (site F) was 5'TCAACTGCAACTCCAAAG3' and for nt16311 (site B) was 5'CCCTTAACAGTACATAG3'.

Statistical analysis was carried out using $\chi^{2}$ testing on data entered on Excel spreadsheets.

Table 1 Polymorphisms discussed in this paper coded for clarity

\begin{tabular}{lllc}
\hline $\begin{array}{l}\text { Location relative } \\
\text { to CRS }\end{array}$ & Base change & $\begin{array}{l}\text { Code used in this } \\
\text { paper }\end{array}$ & Frequency \\
\hline 16069 & C to T & D & $1 / 20$ \\
16093 & T to C & C & $1 / 20$ \\
16189 & T to C & E & $1 / 20$ \\
16224 & T to C & A & $15 / 20$ \\
16256 & C to T & F & $1 / 20$ \\
16311 & T to C & B & $5 / 20$ \\
310 tract & Del C & Short & \\
& No change & Medium & \\
& Ins C & Long & \\
\hline
\end{tabular}




\section{Results}

Sequence variation in DNA samples from mothers and offspring

Sequence analysis of the first hypervariable region (HV1) of the large noncoding region was carried out in parental DNA from 18 trios comprising mother, father and placenta. In four cases, paternity was confirmed by analysis of minisatellite nuclear DNA (University Diagnostics Ltd, Teddington, Middlesex, UK). In 11/18 families, there were sequence differences between mother and father at either 16224 and/or $16311 \mathrm{bp}$ (henceforth sites A and B). Heteroplasmy was apparent and these two polymorphisms were therefore studied in more detail using solid-phase minisequencing, along with another mother-placental pair (P1). Using this method, we found heteroplasmy in $16 / 18(89 \%)$ at $\mathrm{A}$ and $7 / 15(47 \%)$ at B of mother-placenta pairs. We refer to the sequence read from the mother's chromogram as the 'major' type, and the heteroplasmic mutant as the 'minor' type. Levels of the minor mtDNA type ranged from 1 to $35 \%$ (means 9.4 and $9.9 \%$, medians 6 and $12 \%$ in maternal and placental DNA, respectively) but were low in most cases. In only $3 / 36$ of the two polymorphic sites (8.3\%) were maternal levels of the variant between 10 and $90 \%$ and at only $2 / 36(5 \%)$ of maternal sites was the level of the minor variant $30 \%$ or above. The mean level of the minor species was very similar in mother's buccal DNA and placenta (Figure 1a). In addition, in one case (P1) we studied an additional heteroplasmic site at T16093C (site C) in multiple samples (see below). T-PCR of a more limited region of HV2 of the large noncoding region in the 16 samples of fetal placenta revealed heteroplasmy for the homopolymeric $\mathrm{C}$ tract surrounding $310 \mathrm{bp}$ in one (patient P2). Because obvious heteroplasmy was apparent in this single case, multiple samples from P2 were studied in more detail (see below). We refer to three different length variants at this site as long, medium and short (Table 1).

In addition, we found heteroplasmic mtDNA polymorphisms in eight of the placental samples from the 13 pregnancies in which we also had cord blood (thus fetal DNA). As well as A and B this included 16069, 16189 and 16256 (sites D, E and F, respectively, Table 1 and Figure 1b).

The level of heteroplasmy was very similar in mother, child and placenta

The level of heteroplasmy at sites A, B and D-F was assessed in 13 individuals from whom cord blood was available using solid-phase mini-sequencing. There is a close correlation between the load of heteroplasmic variants between cord blood and placenta (Figure $1 \mathrm{~b}$ ) at one site (including $\mathrm{A}, \mathrm{B}$ and $\mathrm{D}-\mathrm{F}$ ) in each of 13 pairs $\left(r^{2}=0.99\right)$. It was similar in maternal mtDNA (buccal or blood) and placenta at sites A and B. Figure 1a shows that even though the percentage of heteroplasmy varied
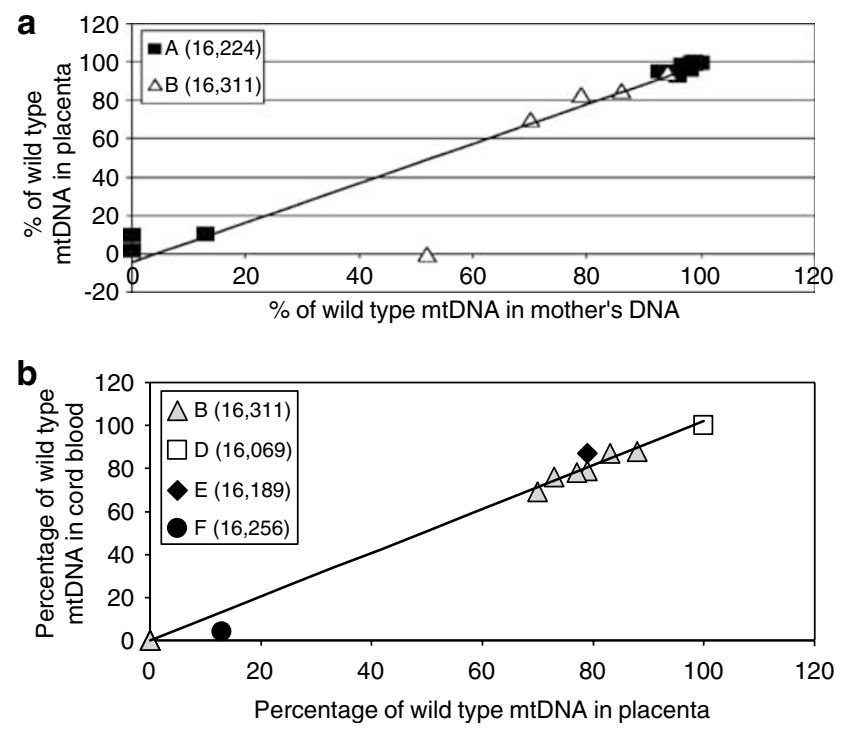

Figure 1 (a) The load of heteroplasmic variant is almost identical in mother and placenta in 15 heteroplasmic mother-placenta pairs at sites $\mathrm{A}$ and $\mathrm{B}$ (Table $1, R^{2}=0.96$ ). (b) The load of heteroplasmic variant is almost identical in placenta and cord blood in the 13 placenta-cord blood pairs at five different sites $\left(R^{2}=0.98\right)$. In each case, the percentage plotted is the proportion of mtDNA in each individual with the Cambridge Reference Sequence ${ }^{50}$ at that location.

between 1 and 35\%, there was a close correlation between the load in mother and placenta, with one outlier $\left(r^{2}=0.96\right)$

\section{Intraplacental distribution of heteroplasmic variants} (multiple samples from each placenta)

Multiple samples were taken from two placentas (16 from term placenta, P1 and 12 from 7-week placenta, P2). One, placenta P1 was heteroplasmic for three different polymorphisms in all of the 16 samples taken, averaging $10.3 \%$ at $\mathrm{A}, 5.7 \%$ at $\mathrm{B}$ and $0.3 \%$ at $\mathrm{C}$. This was very comparable to levels in maternal blood at $11.6,5.9$ and $0.43 \%$, respectively (Table 2). The distribution of these mtDNA polymorphisms in different samples from the same placenta is shown in Figure 2b. No cosegregation of polymorphisms is apparent and there was no association of variant load with region of the placenta or layer from which the sample was taken (not shown). In the case of variant A levels ranged from 0 to $19.6 \%$, but there was less variation for B and C (Figure 2b, top panel). Similarly, (P2) placenta from a 7-week termination of pregnancy was heteroplasmic for $\mathrm{C}$ insertion polymorphisms within the homopolymeric $\mathrm{C}$ tract (ie the run of $\mathrm{C}$ residues between 303 and $315 \mathrm{bp}$ ). This generates nested heteroplasmic length variants ${ }^{20}$ (Figure 2a, panel 1; henceforth long, short and medium variants). Quantitation of the proportion of each length variant in 12 different samples of P2, using T-PCR, demonstrated variation in the distribution of 
Table 2 The mean level of polymorphic variant in 16 placental samples from patient P1 reflected the level in mother's blood

\begin{tabular}{lccrr}
\hline Polymorphism & Mothers level of polymorphism (\%) & Mean level in placental samples (\%) & Standard deviation of mean & Range (\%) \\
\hline A (C16224T) & 11.6 & 10.3 & 5.36 & $0-19.6$ \\
B (T16311C) & 5.9 & 5.7 & 2.70 & $0.6-11.4$ \\
C (T16093C) & 0.4 & 0.3 & 0.09 & $0.18-0.54$ \\
\hline
\end{tabular}

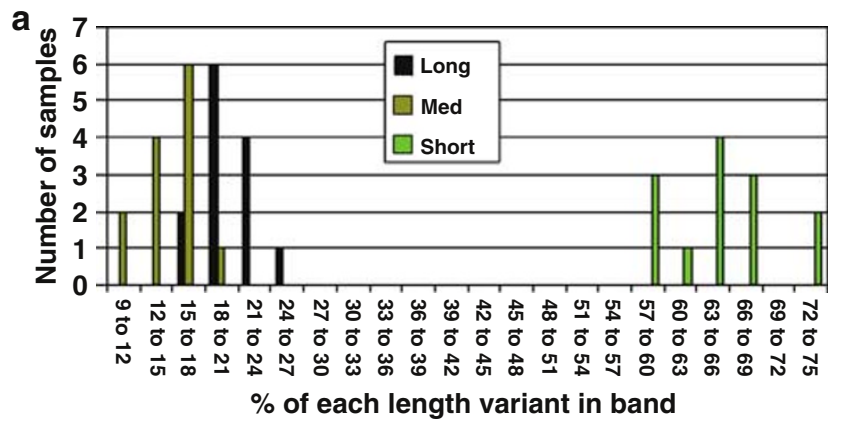

b
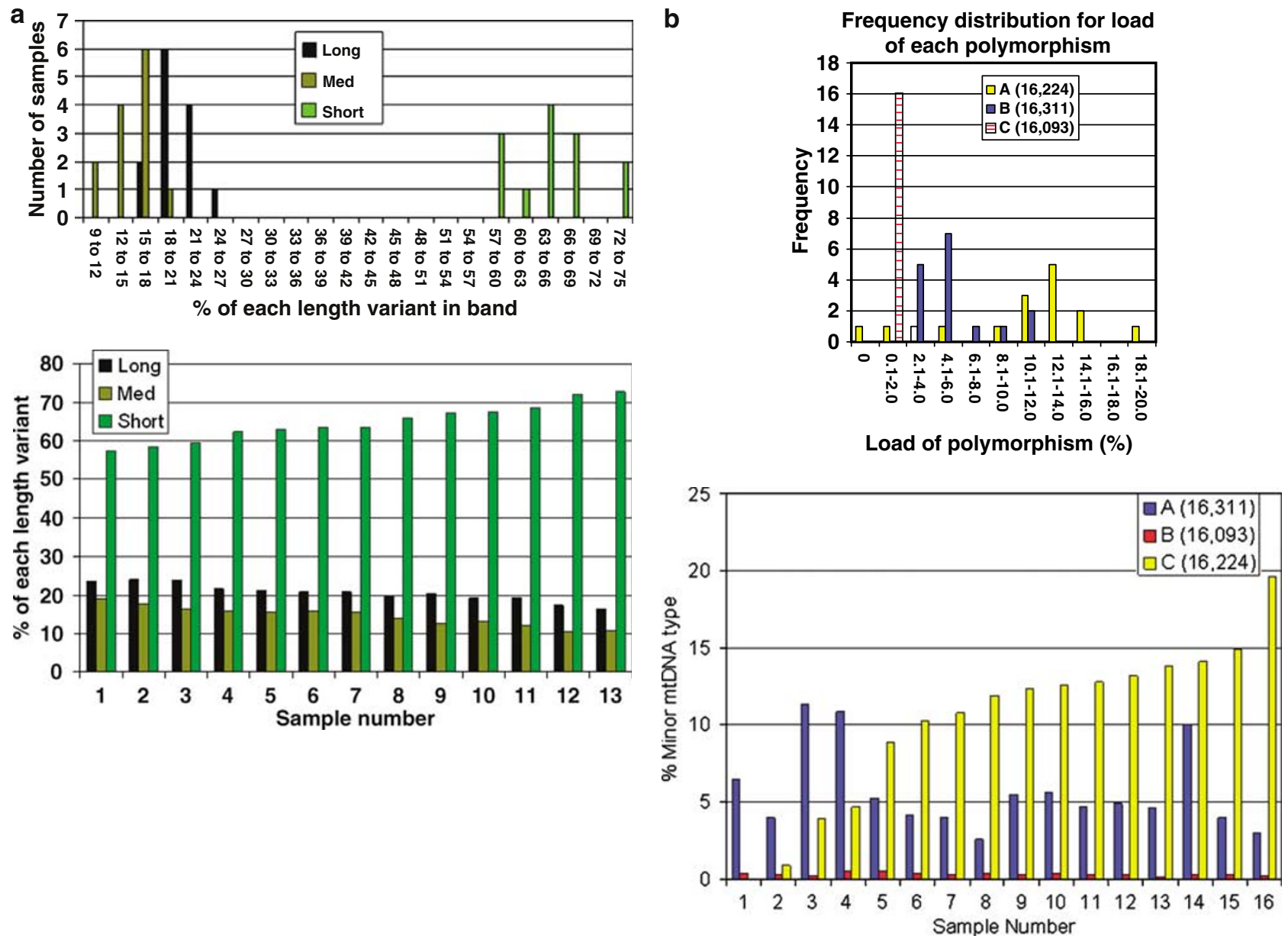

Figure 2 (a) T-PCR was used to quantitate the proportion of three mtDNA length variants in endometrium (sample 1) and in 12 samples of a single 7-week placenta (samples 2-13). The proportion of each length variant is plotted in two ways, by frequency of each samples containing particular proportions (top panel) and by distribution of length variants in each sample (lower panel). The shortest variant appears to be the major type. (b) Solid-phase minisequencing was used to quantitate the proportion of three mtDNA polymorphic variants (point mutations A, B and C, Table 1) in 16 samples from a term placenta. Panel 1 is a bar chart showing the frequency of samples containing different levels of mutant, panel 2 is a bar chart showing the level of each of the three mutants in the 16 different samples (the location from which the sample was taken are: umbilical cord (1, 10), other placental vessel (2), blood clot (four from umbilical vessel, 11), maternal surface $(3,5,7,12,15)$, deep $(6,8,16)$, membranes $(9,13,14)$ ).

length variants in all regions (Figure 2a, panel 2). The variation may be more marked in the term placenta than in the 7-week placenta (coefficients of variation $0.27-0.51$ and $0.07-0.18$, respectively), although this could be because the polymorphisms were different in the two cases.
The number of adult individuals with the $A$ and $B$ (C16 224T and T16311C) polymorphisms suggests a founder effect

If the mutation rate of two polymorphisms is sufficiently high, they may appear to segregate independently. The A and $\mathrm{B}$ polymorphisms were present in 30 and $38 \%$ of all 
Table 3 The number (percent) of adult individuals with the A and B polymorphisms suggests a founder effect

\begin{tabular}{lccc}
\hline & Polymorphism A 16 224C (WT) & Polymorphism A 16 224T (variant) & Total \\
\hline Polymorphism B 16311T (WT) & $23(57.5)$ & $2(5.0)$ & $25(62.5)$ \\
Polymorphism B 16311C (variant) & $5(12.5)$ & $10(25.0)$ & $15(37.5)$ \\
Total & $28(70.0)$ & $12(30.0)$ & $40(100.0)$ \\
\hline
\end{tabular}

parental haplotypes. They tended to occur together, rather than on their own (Table 3). It is already known that this combination of polymorphisms is frequent in particular mitochondrial lineages (such as haplogroup $\mathrm{K}^{21}$ ). $\chi^{2}$ testing (Table 3) showed that there was a marked deficiency of single occurrences that would be expected in a purely random distribution $(P<0.001)$.

\section{Discussion}

The use of naturally occurring polymorphisms along with sensitive detection methods enabled us to study a much larger group of mother-child pairs than if we had confined our studies to pathogenic variants. ${ }^{22}$ In control placentas, we have demonstrated that the level of polymorphic heteroplasmic mtDNA variants is very similar in mother, cord blood and placenta. We present the first evidence for mtDNA segregation in placenta, but this may be apparent only where placental samples are very small $(<10 \mathrm{mg})$. This information is important for prenatal diagnosis of mtDNA diseases.

We identified heteroplasmy in a high proportion of maternal samples, but only $3 / 36$ of these $(8.3 \%)$ had levels between 10 and $90 \%$ and at only $2 / 36$ (5\%) of maternal sites was the level $30 \%$ or above. It is thus unlikely that the heteroplasmy we documented in the remaining samples would have been detected by simply inspecting chromograms without focusing on these specific sites. Previous reports that have used chromograms as the means of detection have thus documented lower frequencies of heteroplasmy, but using sensitive methods these can be as high as on $13.8 \%{ }^{17}$ of blood samples at sites within HV1. Consistent with our results, these studies commented on heteroplasmy being a notable occurrence at $16311 \mathrm{bp}$ (site B) ${ }^{23}$ and at $16093 \mathrm{bp}$ (site D) where it reached a population frequency of $5 \%{ }^{17}$

Levels of heteroplasmic variant were almost identical in placental samples and cord blood $\left(r^{2}=0.99\right)$, although cord blood was only available on 13 samples. This is consistent with the limited information on pathogenic mutations, which suggest that the level of mutant in blood at birth may reflect the load in other tissues, ${ }^{24}$ even though some mutants develop a very variable tissue distribution postnatally. ${ }^{25,26}$ Taken together with published data, ${ }^{7,22,27-30}$ these findings suggest that the level of polymorphic mtDNA variant in these placental samples probably closely reflects the load in cord blood, and potentially the load in the baby. This is a very important precondition for prenatal diagnosis of mtDNA diseases.

Heteroplasmy was very common in the polymorphic sites investigated here. The high frequency of heteroplasmy would be consistent with either a very high rate of somatic mutation or with heteroplasmy that has been stably inherited from the mother. We are not able to distinguish between these alternatives without further analysis of early embryos or oocytes. However, comparing the parental sequences (Table 3) clearly shows that the polymorphisms A and B (16224C and 16311C) cosegregate in the whole population, that is, there is a founder effect in haplogroup K. A high frequency of heteroplasmy at these sites in the germline would minimize such cosegregation.

If somatic mutation were not an issue, the close correlation between the level of heteroplasmic mtDNA variant in placenta and mother (Figure 1b) would suggest a bottleneck with a large number of segregating units. In only one case was there a discrepancy between the load in mother and placenta, consistent with a tighter mitochondrial bottleneck during oogenesis, but we were unable to estimate the bottleneck size accurately because cord blood was not available. We have previously demonstrated that a major component of the bottleneck has occurred by the time ooctyes are mature ${ }^{12}$ for both pathogenic and polymorphic mtDNA variants. Furthermore, germline segregation is widely held to be random for both pathogenic $^{31}$ and polymorphic ${ }^{32}$ mtDNA variants. Although there is good evidence that segregation of pathogenic mutant mtDNA is often nonrandom in cell lines and in patients from birth onwards, there are no published data on mtDNA segregation between conception and birth. We demonstrated that multiple placental samples from the same individual showed variation from 0 to $19.6 \%$ (Figure 2). This is the first evidence for segregation of mtDNA in placenta, and it is entirely consistent with previous data showing that neighboring regions of placental tissue are arranged in 'patches' or clones, arising from different cells in the early embryo. ${ }^{33}$ The apparent discrepancy between the level of heteroplasmic polymorphism in the single placental samples (Figure 1) and the multiple samples (Figure 2) may depend on the size of the sample. In the majority of motherplacenta pairs, we took a single sample of placenta, which was substantially larger $(50-100 \mathrm{mg}$ of tissue) than the multiple samples taken from the two placentas studied in 
detail (5-10 mg). The latter are comparable in size to normal CVS (10-30 mg, P Chamberlain, personal communication). The larger 50-100 mg fragments are presumably more likely to sample two or more placental patches than the smaller fragments. Our data suggest that sample variation attributable to patch size is more marked in the term placenta, where patches will presumably have grown larger than they are in the 7-week placenta. However, it might equally be explained by the difference in type of mutation that we used (length variation in the 7-week and point mutations in the term placenta) and further work is needed to clarify this. In practice, the magnitude of the variation that we found in 7-week placenta may be too small to have much clinical impact (coefficient of variation $<0.18$ ). We suggest that accurate prenatal diagnosis for some mtDNA diseases may require at least two separate samples. Although it is not clear whether and by how much taking two samples would impair fetal viability, this might increase the miscarriage rate. ${ }^{34}$ We are currently investigating the feasibility of preimplantation genetic diagnosis (PGD) for mtDNA disease and intend to recommend concordance testing of two blastomeres for embryos to be implanted in the uterus.

Studies of human placental clonality have usually focused on confined placental mosaicism (CPM), detected in $2 \%$ of all pregnancies. CPM refers to the presence of two karyotypically different cell lines in the placenta with only one in the fetus. ${ }^{33}$ As zones derived from different nuclear gene founders correspond to clones of cells in the early embryo, one would predict that they also represent zones for mitochondrial founders. It would be interesting to establish whether this is indeed the case.

Animal studies have not examined mtDNA segregation in extra-embryonic structures in detail. There are a small number of studies of polymorphic mtDNA variants on embryos where heteroplasmy has been generated artificially by manipulation of early embryos from different polymorphic mouse strains. These are consistent with a uniform distribution throughout both the preimplantation $^{35,36}$ and newborn embryo. ${ }^{10,32,37,38}$

Similarly, studies of pathogenic variants in human placenta have been very limited. In one case, the proportion of mutant was similar to but slightly higher than that of the child. ${ }^{26}$ In most genetic disorders, amniocyte culture can be used when the CVS is too small for reliable analysis, but for mtDNA disorders such analyses are complicated by the possibility that mutant mtDNA can be lost with sequential culture. Prenatal diagnosis of T8993G and T8993A disease (associated with Maternally Inherited Leigh's Syndrome for MILS) has demonstrated a close association between the level of mutant in CVS and neonate and/or extra-embryonic structures. ${ }^{7}$ Apart from this group, there are very few published studies of CVS in mtDNA disease. However, it is unlikely that CVS could be absolutely reliable because it is certain that paternal inheritance of mtDNA has occurred in at least one case. ${ }^{8}$ However, this occurrence must be rare on a population basis because the only other reports ${ }^{39}$ are in abnormal embryos with arrested development. ${ }^{40}$ We and others have previously been unable to detect significant levels of paternal mtDNA polymorphisms in placenta, even in ICSI where the normal processes governing its elimination might be disrupted. ${ }^{19,41,42}$ In the single case report of paternally inherited mtDNA the authors identified mtDNA recombinants using PCR analysis of muscle mtDNA. ${ }^{43}$ If this were a common occurrence that involved the germline it should be evident in population studies, and currently there is little or no clear evidence that this is the case. ${ }^{44,45}$ It is therefore likely to be extremely rare. Therefore, the heteroplasmy that we found in placenta is unlikely to be of paternal origin.

In conclusion, we present the first evidence for mtDNA segregation in placenta. This suggests that the mtDNA in a single CVS of $10 \mathrm{mg}$ may be unrepresentative of the whole placenta. Large or duplicate samples may be necessary for reliable prenatal diagnosis of mtDNA disease. Although our studies were confined to polymorphic mtDNA variants, there is no evidence to suggest that there is a systematic difference between pathogenic mutants and polymorphisms prenatally. ${ }^{12,31,32}$ There was a close correlation of variant load in maternal, fetal blood and placental mtDNA. Provided that segregation of neutral and pathogenic mtDNA mutants is similar in utero, this suggests that in most cases placenta does reflect the load of mutant mtDNA in the baby. We therefore anticipate further development of prenatal diagnosis of mtDNA diseases using CVS and PGD. Nuclear transfer $^{15}$ remains controversial ${ }^{46-49}$ but may ultimately be integrated into genetic management. Further studies to confirm that the levels of both polymorphic and pathogenic mtDNA mutants in CVS are also representative of those in the offspring are essential.

\section{Acknowledgements}

We are very grateful to the patients and their clinicians for providing samples, to Drs K Morten, S Kennedy, P Chamberlain, $P$ and $R$ Boyd, $H$. Stewart and $\mathrm{Mr}$ A Balen for useful discussions, and Professors $L$ Bindoff and $C$ Redman for critical reading of the manuscript. This work was supported by Wellcome Trust Project grant and by Action Medical Research. Jo Poulton was a Royal Society URF and Martin Scott-Brown a Royal Society summer student. There are no competing interests.

\section{References}

1 Lightowlers R, Chinnery P, Turnbull D, Howell N: Mammalian mitochondrial genetics: hereditary, heteroplasmy and disease. Trends Genet 1997; 13: 450-455.

2 Poulton J, O'Rahilly S, Morten K, Clark A: Mitochondrial DNA, diabetes and pancreatic pathology in Kearns-Sayre syndrome. Diabetologia 1995; 38: 868-871. 
3 Weber K, Wilson J, Taylor L, Brierley E, Johnson M, Turnbull D: A new mtDNA mutation showing accumulation with time and restriction to skeletal muscle. Am J Hum Genet 1997; 60: 373-380.

4 Larsson NG, Holme E, Kristiansson B, Oldfors A, Tulinius M: Progressive increase of the mutated mitochondrial DNA fraction in Kearns-Sayre syndrome. Pediatr Res 1990; 28: 131-136.

5 Poulton JMA, Pike M, Seller A, Marchington DR, Kennedy S, Brown GK: Advances in genetic management of patients with mitochondrial disease. Dev Med Ch Neurol 2001; 42: 6.

6 Amiel J, Gigarel N, Benacki A et al: Prenatal diagnosis of respiratory chain deficiency by direct mutation screening. Prenat Diagn 2001; 21: 602-604.

7 White S, Collins V, R RW et al: Genetic counseling and prenatal diagnosis for the mitochondrial DNA mutations at nucleotide 8993. Am J Hum Genet 1999; 65: 474-482.

8 Schwartz M, Vissing J: Paternal inheritance of mitochondrial DNA. N Engl J Med 2002; 347: 576-580.

9 Larsson NG, Tulinius $\mathrm{MH}$, Holme E et al: Segregation and manifestations of the mtDNA tRNA(Lys) $A \rightarrow G(8344)$ mutation of myoclonus epilepsy and ragged-red fibers (MERRF) syndrome. Am J Hum Genet 1992; 51: 1201-1212.

10 Chinnery PF, Thorburn DR, Samuels DC et al: The inheritance of mitochondrial DNA heteroplasmy: random drift, selection or both? Trends Genet 2000; 16: 500-505.

11 Marchington D, Hartshorne G, Barlow D, Poulton J: Homoploymeric tract heteroplasmy in mtDNA from tissues and single oocytes: support for a genetic bottleneck. Am J Hum Genet 1997; 60: $408-416$.

12 Marchington DR, Macaulay V, Hartshorne GM, Barlow D, Poulton J: Evidence from human oocytes for a genetic bottleneck in an mtDNA disease. Am J Hum Genet 1998; 63: 769-775.

13 Blok R, Cook D, Thorburn D, Dahl H: Skewed segregation of the mtDNA nt $8993(\mathrm{~T} \rightarrow \mathrm{G})$ mutation in human ocytes. Am J Hum Genet 1997; 60: 1495-1501.

14 Marchington JPaD: Progress in Genetic counselling and prenatal diagnosis of maternally inherited mtDNA diseases. Neuromusc Disord 2000; 10: 484-487.

15 Taylor RW, Turnbull DM: Mitochondrial DNA mutations in human disease. Nat Rev Genet 2005; 6: 389-402.

16 Brown DT, Samuels DC, Michael EM, Turnbull DM, Chinnery PF: Random genetic drift determines the level of mutant mtDNA in human primary oocytes. Am J Hum Genet 2001; 68: 533-536.

17 Tully LA, Parsons TJ, Steighner RJ, Holland MM, Marino MA, Prenger VL: A sensitive denaturing gradient-Gel electrophoresis assay reveals a high frequency of heteroplasmy in hypervariable region 1 of the human mtDNA control region. Am J Hum Genet 2000; 67: 432-443.

18 Stoneking M: Hypervariable sites in the mtDNA control region are mutational hotspots. Am J Hum Genet 2000; 67: 1029-1032.

19 Marchington DR, Scott Brown MS, Lamb VK et al: No evidence for paternal mtDNA transmission to offspring or extra-embryonic tissues after ICSI. Mol Hum Reprod 2002; 8: 1046-1049.

20 Cavelier L, Jazin E, Jalonen P, Gyllensten U: MtDNA substitution rate and segregation of heteroplasmy in coding and noncoding regions. Hum Genet 2000; 107: 45-50.

21 Richards MB, Macaulay VA, Bandelt HJ, Sykes BC: Phylogeography of mitochondrial DNA in western Europe. Ann Hum Genet 1998; 62: 241-260.

22 White SL, Shanske S, Biros I et al: Two cases of prenatal analysis for the pathogenic $T$ to $G$ substitution at nucleotide 8993 in mitochondrial DNA. Prenat Diagn 1999; 19: 1165-1168.

23 Tagliabracci A, Turchi C, Buscemi L, Sassaroli C: Polymorphism of the mitochondrial DNA control region in Italians. Int J Legal Med 2001; 114: 224-228.

24 Rahman S, Poulton J, Marchington D, Suomalainen A: Decrease of $3243 \mathrm{~A} \rightarrow \mathrm{G}$ mtDNA mutation from blood in MELAS syndrome: a longitudinal study. Am J Hum Genet 2001; 68: 238-240.

25 McShane MA, Hammans SR, Sweeney M et al: Pearson syndrome and mitochondrial encephalomyopathy in a patient with a deletion of mtDNA. Am J Hum Genet 1991; 48: 39-42.
26 Suomalainen A, Majander A, Pihko H, Peltonen L, Syvanen AC: Quantification of tRNA3243(Leu) point mutation of mitochondrial DNA in MELAS patients and its effects on mitochondrial transcription. Hum Mol Genet 1993; 2: 525-534.

27 Bartley J, Senadheera D, Park P, Brar H, Abad D, Wong L-J: Prenatal diagnosis of T8993G mitochondrial DNA point mutation in amniocytes by heteroplasmy detection. Am J Hum Genet 1996; 59: S1839.

28 Harding AE, Holt IJ, Sweeney MG, Brockington M, Davis MB: Prenatal diagnosis of mitochondrial DNA ${ }^{8993 T-G}$ disease. Am J Hum Genet 1992; 50: 629-633.

29 Thorburn DR, Dahl HH: Mitochondrial disorders: genetics, counseling, prenatal diagnosis and reproductive options. Am J Med Genet 2001; 106: 102-114.

30 Chou YJ, Ou CY, Hsu TY et al: Prenatal diagnosis of a fetus harboring an intermediate load of the A3243G mtDNA mutation in a maternal carrier diagnosed with MELAS syndrome. Prenat Diagn 2004; 24: 367-370.

31 Brown DTSD, Michael EM, Turnbull DM, Chinnery PF: Random genetic drift determines the level of mutant mtDNA in human primary oocytes. Am J Hum Genet 2001; 68: 533-536.

32 Jenuth J, Peterson A, Fu K, Shoubridge E: Random genetic drift in the female germline explains the rapid segregation of mammalian mitochondrial DNA. Nat Genet 1996; 14: 146-151.

33 Lau AW, Brown CJ, Penaherrera M, Langlois S, Kalousek DK, Robinson WP: Skewed X-chromosome inactivation is common in fetuses or newborns associated with confined placental mosaicism. Am J Hum Genet 1997; 61: 1353-1361.

34 Rhoads GG, Jackson LG, Schlesselman SE et al: The safety and efficacy of chorionic villus sampling for early prenatal diagnosis of cytogenetic abnormalities. N Engl J Med 1989; 320: 609-617.

35 Meirelles F, Smith L: Mitochondrial genotype segregation during preimplantation development in mouse heteroplasmic embryos. Genetics 1998; 148: 877-883.

36 Molnar M, Shoubridge E: Preimplantation diagnosis for mitochondrial disorders. Neuromusc Disord 1999; 9: 521.

37 Meirelles F, Smith L: Mitochondrial genotype in a mouse heteroplasmic lineage produced by embryonic karyoplast transplantation. Genetics 1997; 145: 445-451.

38 White S: Molecular Mechanisms of Mitochondrial Disorders: The Murdoch Institute. Melbourne: University of Melbourne, 1999, p 282.

39 Filosto M, Mancuso M, Vives-Bauza C et al: Lack of paternal inheritance of muscle mitochondrial DNA in sporadic mitochondrial myopathies. Ann Neurol 2003; 54: 524-526.

40 St John JSD, Dimitriadi K, Barnes A: Failure of elimination of paternal mitochondrial DNA in abnormal embryos. Lancet 2000; 355: 200.

41 Houshmand M, Holme E, Hanson C, Wennerholm UB, Hamberger L: Is paternal mitochondrial DNA transferred to the offspring following intracytoplasmic sperm injection? J Assist Reprod Genet 1997; 14: 223-227.

42 Danan CSD, Van Steirteghem A, Cazeneuve C: Evaluation of parental mitochondrial inheritance in neonates born after intracytoplasmic sperm injection. Am J Hum Genet 1999; 65: $463-473$.

43 Kraytsberg Y, Schwartz M, Brown TA et al: Recombination of human mitochondrial DNA. Science 2004; 304: 981.

44 Piganeau G, Eyre-Walker A: A reanalysis of the indirect evidence for recombination in human mitochondrial DNA. Heredity 2004; 92: $282-288$.

45 Hagelberg E: Recombination or mutation rate heterogeneity? Implications for mitochondrial eve. Trends Genet 2003; 19 $84-90$.

46 Sun YH, Chen SP, Wang YP, Hu W, Zhu ZY: Cytoplasmic impact on cross-genus cloned fish derived from transgenic common carp (Cyprinus carpio) nuclei and goldfish (Carassius auratus) enucleated eggs. Biol Reprod 2005; 72: 510-515.

47 Hiendleder S, Prelle K, Bruggerhoff K et al: Nuclear-cytoplasmic interactions affect in utero developmental capacity, phenotype, 
and cellular metabolism of bovine nuclear transfer fetuses. Biol Reprod 2004; 70: 1196-1205.

48 Brenner CA, Kubisch HM, Pierce KE: Role of the mitochondrial genome in assisted reproductive technologies and embryonic stem cell-based therapeutic cloning. Reprod Fertil Dev 2004; 16: $743-751$
49 St John JC, Schatten G: Paternal mitochondrial DNA transmission during nonhuman primate nuclear transfer. Genetics 2004; 167: 897-905.

50 Anderson S, Bankier AT, Barrell BG et al: Sequence and organisation of the human mitochondrial genome. Nature 1981; 290: 457-465. 